\title{
Social Serendipity: Mobilizing Social Software
}

\author{
A new mobile-phone-based system uses Bluetooth hardware addresses \\ and a database of user profiles to cue informal, face-to-face interactions \\ between nearby users who don't know each other, but probably should.
}

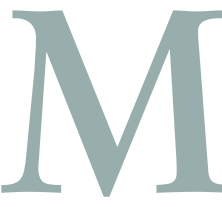

obile phones have been adopted faster than any technology in human history and are now available to the majority of people on Earth who earn more than US\$5 a day. More than 600 million phones were sold in 2004, many times more than the number of personal computers sold that year. ${ }^{1}$ This new infrastructure of phones is ripe for novel applications, especially given continual increases in their processing power.

Many mobile devices also incorporate lowpower wireless connectivity protocols, such as Bluetooth, that can be used to identify an individual to other people nearby. We have developed an architecture that leverages this functionality in mobile phones-originally designed for communication at a distance- - to connect people across the room. Serendipity is an application of the architecture. It combines the existing communications infrastructure with online introduction systems' functionality to facilitate interactions between physically proximate people through a centralized server.

\section{Bluetooth proximity detection}

After years of hype, Bluetooth is finally seeing mass-market adoption in mobile electronics. According to the official Bluetooth Web site (www.bluetooth.com/news/releases.asp), more than three million Bluetooth devices are currently sold each week. The protocol's primary use is to connect items such as a wireless headset or laptop to a phone. As a by-product, Bluetooth devices can also discover other devices nearby. This "accidental" functionality enables mobile communication devices to act as a gateway to online introduction systems, such as Friendster, Monster, or Match.com. The difference is that mobile devices situate the introduction in an immediate social context, rather than asynchronously in front of a desktop computer.

\section{BlueAware}

We designed a Mobile Information Device Profile (MIDP) 2.0 application, called BlueAware, that can run passively in the background on many of today's Bluetooth phones. This social-scanning application capitalizes on a unique Bluetooth identifier (BTID) number that mobile phones with Bluetooth personal-area-network capabilities transmit when queried.

In a proximity $\log$, BlueAware records and timestamps the BTIDs it encounters. If it detects a device that it has not recently recorded in the proximity $\log$, it automatically sends the discovered BTID to the Serendipity server.

Privacy-driven features. When a user turns on a BlueAware-equipped phone, the application begins running in the background automatically, alerting the user to its presence with a dialog box 
Figure 1. Methods for detecting Bluetooth devices: (a) BlueAware running in the foreground on a Nokia Series 60 phone, and (b) BlueDar device using a Bluetooth beacon coupled with an 802.11 b WiFi bridge.

at start-up. Additionally, the interface design lets users read and delete the specific data being collected, as Figure 1a shows, or stop the logging completely.

Refresh rate versus battery life. Continually scanning and logging BTIDs can expend the battery of an older mobile phonesay, a two-year-old Nokia 3650-in about 18 hours. While continuous scans provide a rich depiction of a user's dynamic environment, most individuals are used to having phones with standby times exceeding 48 hours. We therefore modified BlueAware to scan the environment only once every five minutes, providing at least 36 hours of standby time.

\section{BlueDar}

Figure $1 \mathrm{~b}$ shows a variation on BlueAware called BlueDar. We developed it to be placed in a social setting and scan continuously for visible devices. BlueDar transmits detected BTIDs to the Serendipity server over an 802.11 b wireless network. It uses a specially designed Bluetooth beacon that incorporates a class 1 Bluetooth chipset controlled by an XPort Web server. ${ }^{2}$ We integrated the beacon with an 802.11 b wireless bridge and packaged the components in an unobtrusive box.

We wrote an application that telnets continuously into multiple BlueDar systems, scans repeatedly for Bluetooth devices, and transmits the discovered BTIDs to our server. Because the Bluetooth chipset is a class 1 device, it can detect any visible Bluetooth device within a working range of about 30 meters.

\section{Serendipity: Situated introductions}

Today's social software isn't very social. From standard customer-rela-

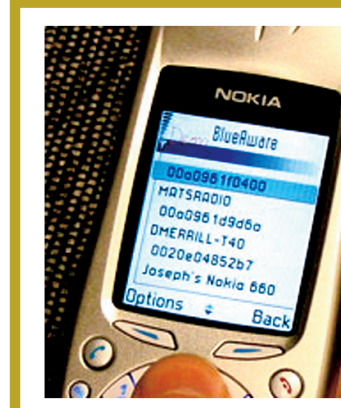

(a)

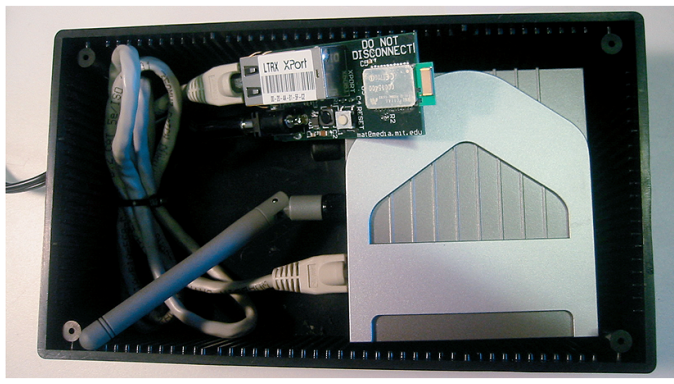

(b) tionship-management systems to Webbased introduction systems, these services require users to be in front of a computer to make new acquaintances. Serendipity, on the other hand, embeds such applications directly into everyday social settings: on the bus, around the water cooler, in a bar, at a conference.

We use desktops, laptops, handheld computers, and mobile phones continually in our work and social lives. These innovations were primarily designed to empower the individual. However, over the past decade, many instantiations of social proximity sensing have appeared for pocket-sized devices (see the sidebar, "Related Work in Mobile Social Software").

\section{Mobile profile matching}

Serendipity's central server contains user profiles along with matchmaking preferences. The profiles are similar to those stored in other social software programs such as LinkedIn and Match.com. However, Serendipity users also provide weights that determine each piece of information's importance when calculating a similarity score. The system calculates a similarity score by extracting the commonalities between two proximate users' profiles and (if available) behavioral data, and summing them according to user-defined weights. If the score is above the threshold set by both users, the server alerts them that someone nearby might interest them.

Users can set thresholds from their phones, along with the weighting schemes to define the similarity metrics.
The user-defined thresholds and weights correspond to profile types such as business networking, bar hopping, and busy modes. A patent on this behavioral/location-based matching and cueing system was filed in early 2004, and is now being licensed from MIT by SenseSix, a company formed to commercialize this technology. ${ }^{3}$

\section{Implementation}

Serendipity receives the BTID and threshold variables from the phones and queries a MySQL database for the user profiles associated with the discovered BTID addresses. If a profile exists, the system calls another script to calculate a similarity score between the two proximate users. When this score is above both users' thresholds, the script sends an alert to their phones with the other user's picture, their commonalities, a list of talking points, and additional contact information (at each user's discretion).

A feedback mechanism lets users rate the introduction message with a number value from 1 to 10 . Currently, only system designers are using this information, but it lays the foundation for a future personalized matchmaking architecture based on collaborative filtering.

\section{Relationship inference}

For approximately 100 users who have opted in, we are also collecting a variety of additional data, including cell tower IDs and communication logs, using the Context application (www. cs.helsinki.fi/group/context). Figure 2 depicts the patterns in cell towers and 


\section{Related Work in Mobile Social Software}

M any applications of social proximity-sensing software have appeared over the past decade. The following projects are by no means a comprehensive review, but rather a sample of the diversity in this burgeoning field.

\section{Lovegety}

Introduced in Japan in early 1998, Lovegety was the first commercial attempt to move introduction systems away from the desktop and into reality. ${ }^{1}$ Users input their responses to a couple of questions into the Lovegety device, which then alerts them when it senses a mutual match nearby.

Gaydar, a similar product specifically targeted for the gay community, appeared in the US soon after Lovegety's launch.

\section{Cell tower/SMS Friend Finders}

Several wireless service providers now offer location-based services to mobile phone subscribers using cell tower IDs. For example, Dodgeball.com users can expose their location to friends by explicitly naming it through their short message service.

\section{Experience Ubicomp Project}

Combining inexpensive radio frequency identification (RFID) tags with traditional conference badges, the Experience Ubicomp Project linked profiles describing many conference presenters with their actual locations. When users would approach a tag reader, a nearby screen would display "talking points" relevant to their interests. ${ }^{2}$

\section{Social Net}

Another project, Social Net, also uses RF-based devices-specifically, the Cybiko wireless communications computer (www.cybiko. com) - to learn proximity patterns between people. When coupled with explicit information about a social network, the device can inform a mutual friend of two proximate people that an introduction might be appropriate. ${ }^{3}$

\section{Hummingbird}

A custom-developed mobile RF device, the Hummingbird, ${ }^{4}$ alerts users when they're near another user. The Hummingbird supports collaboration and was designed to augment traditional office communication media such as instant messaging and email.

\section{Jabberwocky}

A mobile phone application, Jabberwocky, performs repeated Bluetooth scans to develop a sense of an urban landscape. By continually logging nearby phones, the system fosters a sense of the "familiar strangers" that create an urban community. ${ }^{5}$ This functionality distinguishes it from Serendipity, which focuses on introducing people directly.

\section{REFERENCES}

1. Y. Iwatani, "Love: Japanese Style," Wired News, 11 Jun 1998; www. wired.com/news/culture/0,1284,12899,00.html.

2. J.F. McCarthy et al., "Proactive Displays and the Experience UbiComp Project," ACM Siggroup Bull., Dec. 2002, pp. 38-41.

3. M. Terry et al., "Social Net: Using Patterns of Physical Proximity over Time to Infer Shared Interests," Proc. Human Factors in Computing Systems (CHI 2002), ACM Press, 2002, pp. 816-817.

4. L.E. Holmquist, J. Falk, and J. Wigström, "Supporting Group Collaboration with Interpersonal Awareness Devices," J. Personal Technologies, vol. 3, nos. 1-2, 1999, pp. 105-124.

5. E. Paulos and E. Goodman, "The Familiar Stranger: Anxiety, Comfort, and Play in Public Places," Proc. Human Factors in Computing Systems (CHI 2004), ACM Press, 2004, pp. 223-230.
Bluetooth devices over the course of a day. It shows clearly that the user is home from 1 a.m. to 10 a.m., then goes to work. We can see the majority of devices detected during regular office hours, with one exception-a device the user comes into contact with outside the workplace in the evening.

We use information about proximity to other mobile devices to make inferences about a user's social network. Looking at the proximity context between two people can reveal much information about the nature of the relationship. For exam- ple, proximity at 3 p.m. by the coffee machines confers a much different meaning from proximity at 11 p.m. at a local bar. We have trained a Gaussian mixture model to detect proximity patterns between users and then correlate these patterns with relationship types.

The labels for this model came from a survey that all experimental subjects took at the end of two months' data collection. The survey asked whom they spent time with both in and out of the office and whom they considered to be in their circle of friends. We compared these labels with estimated location (using cell tower distribution and static Bluetooth device distribution), proximity (measured from Bluetooth logs), and time of day; we discovered it is possible to identify office acquaintances, outside friends, and people within a circle of friends with good accuracy. ${ }^{4}$

Figure 3 illustrates some of the information that permits inference of friendship. It shows that our sensing technique is picking up the common-sense phenomenon that office acquaintances see each other frequently in the office but 
Figure 2. A sample user's daily distribution of (a) observed cell tower transitions and (b) Bluetooth device detections. The midday "hot spot" occurs when the user is in the office.

rarely outside the office. Conversely, friends often see each other outside the office, even if they are also coworkers.

\section{Other introduction services}

Most interactions instigated by Serendipity are based on similarity scores and information sent to two users. However, proximity Web pages let users make part of their profiles public and view the public information of other nearby users, regardless of similarity scores. With this feature, users need not disclose information about themselves to receive information about others.

We have also enabled a feature that sends only an anonymous text message alerting users that a person who shares similar interests is nearby; both users must respond "yes" to actuate the dissemination of any personal information. This feature preserves user privacy and minimizes disruptions.

Other methods exist for mediating introductions. For example, Social Net relies on a mutual friend to make the introduction (see the sidebar, "Related Work in Mobile Social Software"). Serendipity could incorporate such a method, alerting a mutual friend-rather than the two individuals-when the algorithm finds a match.

\section{User studies}

We have tested and iterated the Serendipity system for almost a year.

Figure 3. Inferring friendship: Contact data from one subject for (a) a friend and (b) an office acquaintance. In each case, the two graphs show the proximity frequency over a single day (top) and a single week (bottom).

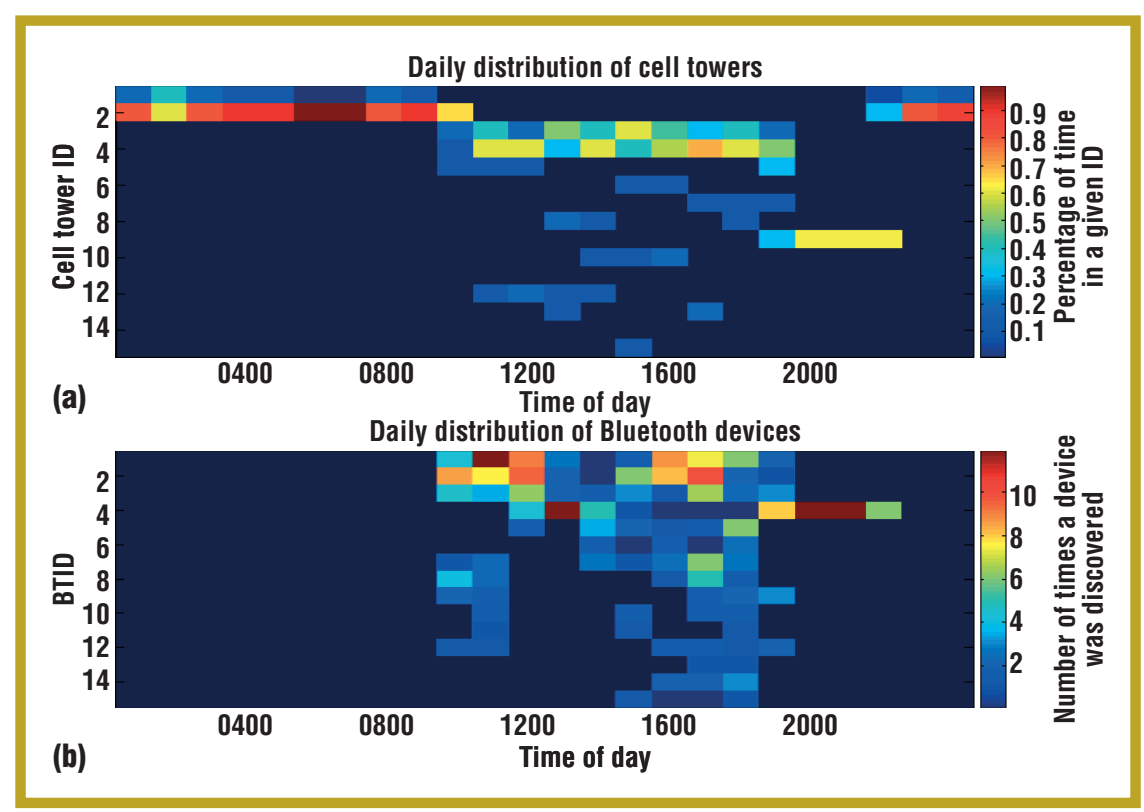

\section{Initial deployment}

In early May 2004, Serendipity had its first test deployment at a conference for senior corporate executives and professors. We created personal profiles for 40 conference participants who picked up their assigned phones upon arrival in the morning. The system supported more than 100 introductions over the course of the day, primarily during the intersession coffee breaks. We used what we learned from this first deployment to refine the system in subsequent versions.

The conference setting necessitated several modifications from our original design. Because all the subjects were proximate to each other during the talks, we had to develop a method for preventing introductions while the talks were in session. Simply hard-coding the conference break schedule into phones was not advisable: first, the duration of the talks was uncertain; second, such an approach would also prevent introductions between people who were outside during a particular talk.

Instead, we used several of our research group's personal Bluetooth devices to pre-

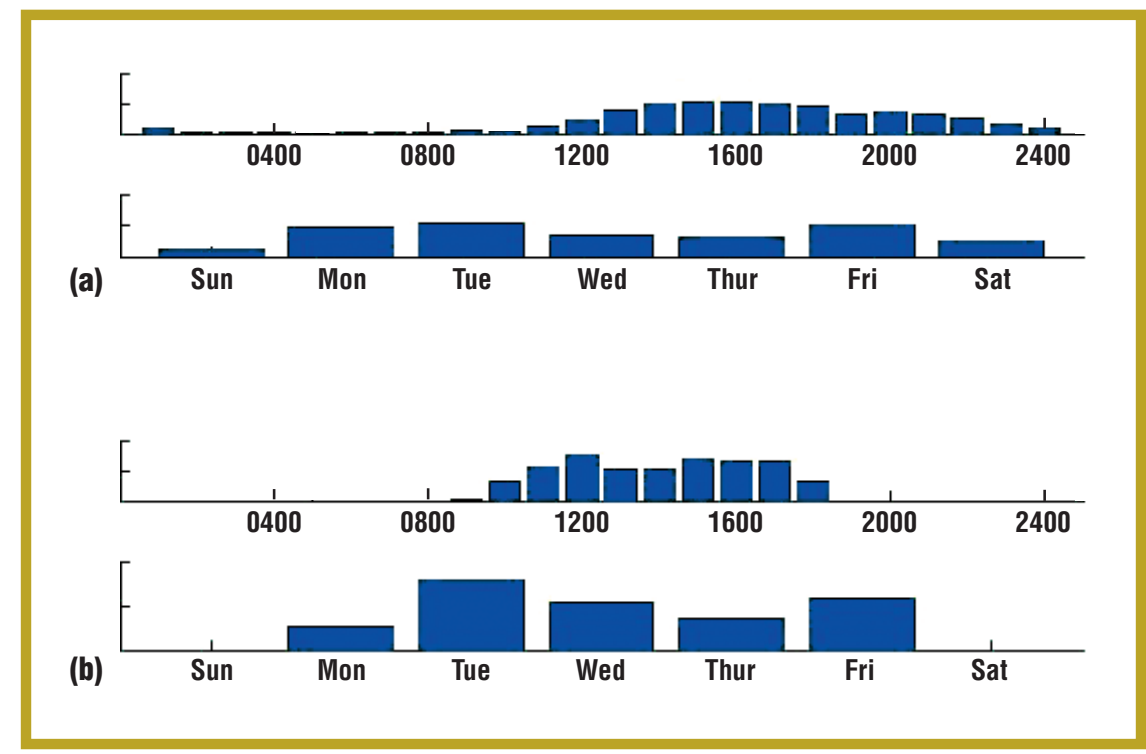




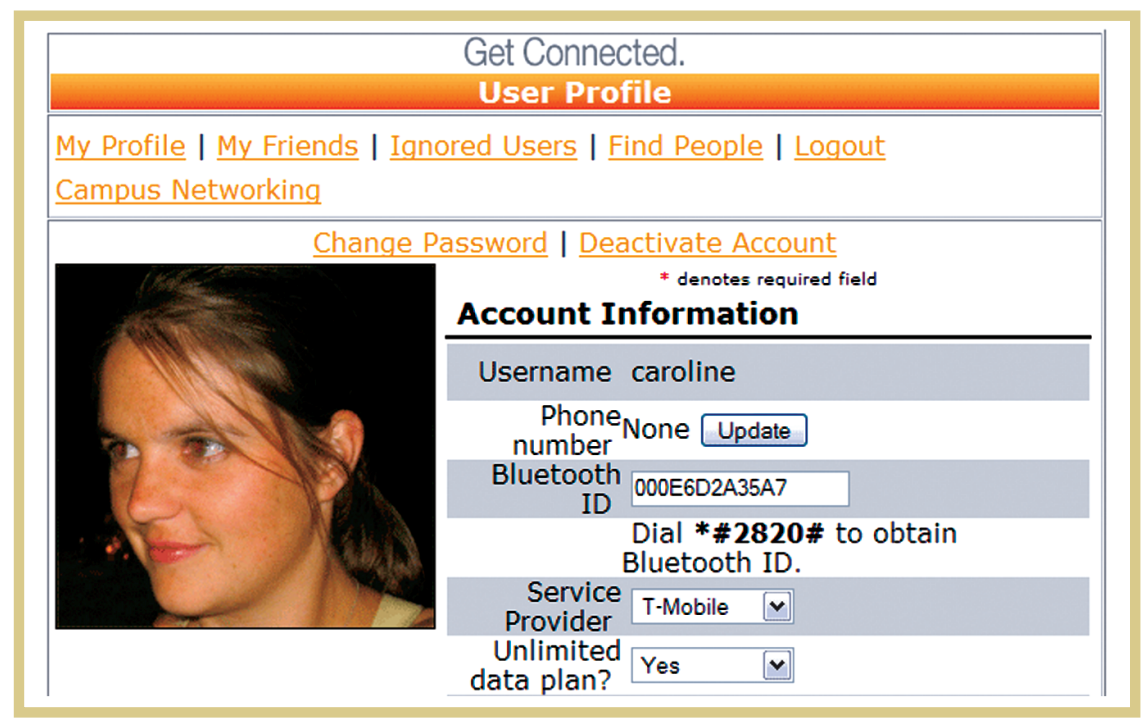

vent intrusions. We had volunteers position themselves throughout the auditorium, each carrying a visible Bluetooth device whose name was changed to Block. Any of the 40 phones inside the auditorium during the talks could detect at least one of these Block devices. When it did so, it paused the application so that the Serendipity system neither recorded information about proximate devices nor sent information to our server.

This succeeded in preventing introductions during talks-when we knew they were inappropriate. However, we had not accounted for the density of people mingling during breaks. Several users complained of receiving multiple introductions within only a few minutes of each other. This led to social disruptions as one conversation just getting underway was interrupted by the initiation of another. One user solved the problem by simply turning his phone off while in conversation and then turning it back on when he was ready to meet someone else. We subsequently formalized this feature as Hidden Mode. We also imposed a maximum on receiving introductions to one every 10 minutes.

Among surprising results was the appreciation expressed by many users who worked for large corporations at being introduced to other coworkers in the same company. A couple of the par- ticipants never understood the application's introduction component; they didn't know what the picture messages about people nearby were meant to accomplish. However, aside from the complaints about disruptive multiple introductions, the initial user feedback was primarily positive.

Few of the initial subjects voiced privacy concerns, although many emphasized the importance of being able to easily turn the application on and off at the user's discretion.

\section{Campus deployment}

Currently Serendipity is running on the phones of 100 users at MIT. Of these, 70 are either students or faculty in the same technical lab, while the remaining 30 are incoming students at the business school adjacent to the laboratory. We are also receiving information from the devices regarding the other users that each user encounters throughout the day. The user profiles from the technical lab are currently bootstrapped from information available within a public project directory. Users can also input personal information and change any aspect of their profile. Figure 4 shows a portion of a user profile.

Early user reactions have been overwhelmingly positive. Engineers and business school students have expressed the greatest enthusiasm for introductions
Figure 4. A small portion of a profile stored on the Serendipity server.

based on their interest in the commercial potential of engineering research projects. The response to introductions between members of the technical lab has also been positive. On average, the lab members are acquainted with only five to seven other subjects in the study. Five percent of the users who received phones elected not to participate; the primary reason related to time- - they didn't want to be interrupted-but two users had privacy concerns as well.

\section{BlueDar deployment}

We have networked BlueDar scanning units in several social settings on campus including the student lounge, near the coffee machines, and at a local bar. Above each device, we put a flyer explaining BlueDar's functionality and the type of data being captured. In this university setting, however, we have found that only one of approximately 150 people (excluding study participants) have a visible Bluetooth device-far fewer than the number of people actually carrying Bluetoothenabled gadgets.

This implies that potential users must decide to make their device visible to participate in the experiment. While some people have expressed reluctance to do so because of concerns about power consumption and security, this remains a viable option to support the matching for many who carry Bluetooth phones that are not MIDP compatible.

\section{Privacy implications}

BlueAware, BlueDar, and Serendipity introduce a significant number of privacy concerns if deployed outside a carefully controlled experiment with human subjects' approval. These privacy issues must be reviewed in detail before releasing this service to the general public.

\section{BlueAware/BlueDar}

All subjects in our experiment have 
explicitly consented to participate, but we are also collecting data about devices carried by people who aren't directly participating in the experiment. However, logging a phone's BTID_for example, 00E6D6602B5-does not expose the an individual's actual identity. Additionally, because the majority of Bluetooth devices are sold with default settings set to "not discoverable," we are operating under the assumption that when a device is consciously turned to "visible" mode, the user knows and accepts that others can detect his or her device.

\section{Serendipity}

Providing a service that supplies nearby strangers with a user's name and picture is rife with liability and privacy issues. The system must be made to ensure that the service never jeopardizes a user's privacy expectations. The measures we have taken to assuage some of these concerns include proximity Web pages, anonymous SMS chat, and the option of limiting interactions to users within a friends-of-friends trust network. Clearly, Serendipity must make as many privacy-protecting tools available as possible to maintain user diversity and, most importantly, keep everyone safe.

\section{Future applications}

Bridging social software introduction systems with current mobile phone technology enables a diverse suite of applications. Conference participants will be able to find the right people during an event. Large companies interested in facilitating internal collaboration could use Serendipity to introduce people who are working on similar projects but who are not within one another's social circles. Individuals could go to a bar and immediately find people with common interests.

\section{Enterprise}

Static employee surveys reflect a severely limited view of an organiza- tion's social network. We propose using proximity data to infer a social network's dynamics. For example, BlueAware data could automatically build a network model of the individuals within an organization and thereby quantify the effects of a management intervention.

Additionally, incorporating Serendipity into the workplace could instigate synergistic collaborations by connecting people who are working on similar material or by connecting a domain

\section{Conferences}

The need for introduction systems at events such as large conferences and trade shows is well established. ${ }^{6}$ Sales people can generate proximity Web pages to present their photos and interests, similar to those that publicize their products and expertise. Conference participants can customize their profiles for connecting only with individuals who match specific interest areas. Our initial deployment showed Serendipity's potential as a conference-networking tool.

\section{Technology-driven societal change is a hallmark of}

\section{our era. A new infrastructure of intelligent mobile devices is influencing culture in unplanned and unprecedented ways.}

expert to employees who are working on a problem in that area. Finally, forming groups based on the individuals' inherent communication behavior rather than a rigid hierarchy might yield significant insights to organizational behavior. We are in discussions now with a large technology company to install several BlueDar units within one of their local campuses and integrate them with an informal knowledge management system. ${ }^{5}$

\section{Dating}

The growth of online dating has soared over recent years as the stigma associated with personal ads diminishes. Serendipity provides users an alternative to encounters with people they've met only through a computer monitor. Although we need many more users than our current sample to test Serendipity's efficacy as a dating tool, we're talking with several online dating companies about the possibility of integrating a similar system in their product lines, which involve millions of active participants.

\section{Beyond Serendipity}

Technology-driven societal change is a hallmark of our era. A new infrastructure of intelligent mobile devices is influencing culture in unplanned and unprecedented ways. For example, SMS text messaging now generates a significant fraction of many service providers' revenues, yet cellular network operators originally developed the protocol as a way for their service technicians to test the network.

Similarly, Serendipity's main use might not involve any of the applications described here but rather something less expected. Perhaps by leveraging trust networks, the system could dramatically change the trade-offs of hitchhiking. Coordinating mobile platforms with embedded computers in cars could facilitate ridesharing and carpooling.

Human-machine interactions. By equipping a physical infrastructure with embedded computing and a Bluetooth transceiver, a variation of the Serendipity 
system could notify human users of nearby resources or facilities. For instance, the system could notify users of a nearby public restroom. If instead of human users, we consider mobile platforms with embedded computers, we can envision other applications. For instance, buses could wait for passengers on a late connection, or delivery vehicles could more efficiently service pickup and drop-off requests.

Role-based access control. RBAC is a technique for assigning user permissions that correspond to an organization's functional roles. ${ }^{7}$ By capturing extensive user behavior patterns over time, our system has the potential to infer not only relationships among users but also their permissions. For example, if two students working in different labs begin collaborating at a coffee shop, they could be granted constrained entrance access to each other's lab.

Public release of Serendipity. Serendipity's final test will be its public launch this summer, under the SenseSix brand, on www.sensesix.com. We hope that the application will prove to be not only robust but also quite popular within the realms we have described as well as those unanticipated.

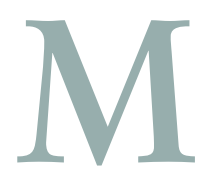
obile phones have become standard attire around the globe. Millions of pockets and purses hold wireless transceivers, microphones, and the computational horsepower of a desktop computer of just a few years ago. Today the majority of this processing power goes unused. This will change, however, when mobile applications shift emphasis toward supporting individual desires to affiliate with other people who share their interests and goals.

Online dating services and knowledge management systems give us glimpses of introduction services, yet the real potential of these new applications will require an infrastructure of socially curious mobile devices that untether social software from the desktop and imbue it into everyday life. We believe the mobile phone market is at a critical tipping point, where functionality will shift from the traditional telephone paradigm to a much broader socio-centric perspective. We hope that this work represents a step in that direction. $\mathbf{P}$

\section{the AUTHORS}

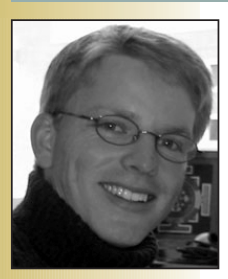

Nathan Eagle is a PhD candidate in media arts and sciences at the MIT Media Laboratory. His research interests include machine learning, mobile computing, and complex networks. He has an MS in management science and engineering and an MS in electrical engineering from Stanford University. Contact him at MIT Media Laboratory, E15-383, 20 Ames St., Cambridge, MA 02139; nathan@media.mit.edu.

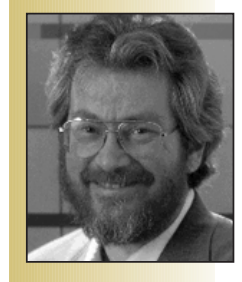

\section{ACKNOWLEDGMENTS}

We are grateful to everyone who participated throughout the application development including Tony Pryor, Pedro Yip, Steve Kannan, Doochan Han, Greg Sterndale, Jon Gips, Mat Laibowitz, Max Van Kleek, and Igor Sylvester. Additionally, we thank members of both Nokia and Nokia Research, particularly Harri Pennanen, Hartti Suomela, Saku Hieta, Peter Wakim, Suvi Hiltunen, and Timo Salomaki.

\section{REFERENCES}

1. B. Wood et al., Market Share: Mobile Terminals, Worldwide, 4Q04 and 2004, market report, Gartner Group, Mar. 2004; http://www.gartner.com/DisplayDocument?id= 473935 .

2. M. Laibowitz, "Parasitic Mobility for Sensate Media," master's thesis, Media Arts and Sciences, Massachusetts Inst. of Technology, 2004.

3. N. Eagle and A. Pentland, "Interaction Cueing Using Wireless Networking and a User Profile Database," MIT case no. 10705T, US patent filed 6 May 2004, Combined Short Radio Network and Cellular Telephone Network for Interpersonal Communications.

4. N. Eagle and A. Pentland, "Reality Mining: Sensing Complex Social Systems," to appear in Personal and Ubiquitous Computing, June 2005.

5. N. Eagle, "Can Serendipity Be Planned?" MIT Sloan Management Rev.. vol. 46, no. 1, Fall 2004, pp 10-14.

6. R. Borovoy et al., "Meme Tags and Community Mirrors: Moving from Conferences to Collaboration," Proc. 1998 ACM Conf. Computer Supported Cooperative Work, ACM Press, 1998, pp. 159-168.

7. R. Sandhu, D. Ferraiolo, and R. Kuhn, "The NIST Model for Role Based Access Control: Towards a Unified Standard," Proc. 5th ACM Workshop on Role Based Access Control, ACM Press, 2000, pp. 47-63.

For more information on this or any other computing topic, please visit our Digital Library at http:// computer.org/publications/dlib. 